\title{
Family consent, communication, and advance directives for cancer disclosure: a Japanese case and discussion
}

Akira Akabayashi, Michael D Fetters and Todd S Elwyn The University of Tokyo, Fapan, The University of Michigan, Ann Arbor, Michigan, USA and the University of Hawaii, Honolulu, Hawaii, USA

\begin{abstract}
The dilemma of whether and how to disclose a diagnosis of cancer or of any other terminal illness continues to be a subject of worldwide interest. We present the case of a 62-year-old fapanese woman afflicted with advanced gall bladder cancer who had previously expressed a preference not to be told a diagnosis of cancer. The treating physician revealed the diagnosis to the family first, and then told the patient: "You don't have any cancer yet, but if we don't treat you, it will progress to a cancer". In our analysis, we examine the role of family consent, communication patterns (including ambiguous disclosure), and advance directives for cancer disclosure in fapan. Finally, we explore the implications for Edmund Pellegrino's proposal of "something close to autonomy" as a universal good. (Fournal of Medical Ethics 1999;25:296-301)
\end{abstract}

Keywords: Family consent; ambiguous disclosure; cancer disclosure; medical ethics; autonomy; informed consent

The dilemma of whether and how to disclose a diagnosis of cancer or of any other terminal illness continues to be a subject of worldwide interest. ${ }^{12}$ In many cultures around the world, the cancer diagnosis is not routinely disclosed to the patient. For example, the practice of nondisclosure is reported to occur in many countries in Eastern and Southern Europe ${ }^{3-6}$; the Middle East $^{7}$; Africa ${ }^{8}$; Asia ${ }^{9-14}$; and other parts of the world ${ }^{8}$ We present an example of what we believe are common communication and decision making patterns for cancer disclosure in one such country, Japan, through the case of a Japanese woman who expressed a preference not to be told a diagnosis of cancer. This case raises a series of important questions regarding cancer disclosure in Japan: 1) What is the historical context of the cancer disclosure debate in Japan? 2) What is the role of family consent for disclosure? 3) What are the patterns of communication relating to ambiguous disclosure and the use of advance directives for disclosure of the cancer diagnosisis and 4) Is the "universal principle of autonomy applicable to this case?

\section{Case presentation}

A 62-year-old Japanese woman presented to के Tokyo hospital with a fever and severe back pain Diagnostic work-up included serological tumoge marker testing and abdominal computed tomography. This revealed advanced gall bladder cances metastatic to the liver and back. Since het expected survival was less than three months an she was not a candidate for surgery or chemo therapy, a regimen of comfort measures and paia control was needed.

The diagnosis was first revealed to her familp members, namely her husband and her son, sep rately from the patient. The husband and son dis cussed it with the daughter, and together the fam ily requested that the patient not be told. Tho family explained that while still healthy the patient had mentioned to them her wish not to be told she developed cancer. This mention of her prefero ence may have been stimulated by intermittent media coverage of the issue in Japan and seeme plausible.

After initial treatment for pain and fever, the

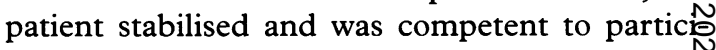
pate in decision making, though she was a littles withdrawn and dependent. The treating physiciar and family met with the patient and in the familye presence, the treating physician told her: "Yo\& don't have any cancer yet, but if we don't treat you, it will progress to a cancer". In response, the patient asked for no further details. An aggressive pain control regimen was continued and thoug $\frac{1}{8}$ she was intermittently drowsy, she died fou months later without apparent suffering from physical pain. The physician never explicit discussed the diagnosis with her. 


\section{Historical context of cancer diagnosis disclosure in Japan}

Although little has been written in English about the history of the cancer disclosure debate in Japan, ${ }^{10}$ scholarly discussion about it can be traced to the late sixties. In 1969, the Japan Society for Cancer Therapy held a panel discussion on "Whether cancer patients should be informed of their disease", during which Matsuoka reported the results of two surveys. In a 1964 survey of 350 Christian Japanese doctors, $16 \%$ of the 132 respondents reported directly telling their patients the cancer diagnosis, while $76 \%$ answered they only told the patient's family the diagnosis. ${ }^{15} \mathrm{~A}$ 1963 survey of 4,300 lay persons in Ehime prefecture revealed that $90 \%$ of respondents desired to be told the diagnosis if they developed cancer.

A substantial body of literature on cancer disclosure in Japan has since appeared, including reports of attitude surveys ${ }^{12}{ }^{16-18}$ and surveys of actual practice. ${ }^{19-22}$ Attitudes of the Japanese public towards cancer disclosure investigated by a nationwide newspaper poll (Yomiuri newspaper) in 1987 revealed that, if afflicted by cancer, $64 \%$ of the subjects would want to be told the diagnosis, $27 \%$ would not, and $9 \%$ replied "other" or did not answer. However, if the patient was a family member of the subject who knew the true diagnosis, only $20 \%$ would tell the patient, $67 \%$ would not tell, and $13 \%$ replied "other" or did not answer. A nationwide survey of family members of deceased cancer patients conducted by the Ministry of Health and Welfare in 1994 showed that $20 \%$ of family members answered that the patients knew their diagnoses because the patients were told the real diagnoses either by family members or doctors, $44 \%$ responded that they thought the patients suspected or knew they had cancer, despite not being told directly, and $29 \%$ answered that they felt the patients did not know their diagnoses at all. ${ }^{23}$

Recognising the growing importance of cancer disclosure as a public health issue, the Ministry of Health and Welfare and the Japan Medical Association published a manual to help guide decision making. ${ }^{24}$ In one key section it states:

"In regard to disclosure of cancer diagnoses, it is not appropriate to tell terminal patients uniformly in all cases. However, the merits of disclosing the terminal diagnosis are significant. It is important to make efforts to disclose the truth while balancing the advantages and disadvantages of disclosure. The patient's age, sex, personality or beliefs, relationship with the family, social status, life experiences, and preferences for terminal care, etc should be considered carefully. The expression and timing of disclosure should be carefully considered; indirect disclosure is also a choice." (Authors' translation)

The manual then lists four important factors which should be taken into account when considering whether to disclose a cancer diagnosis: 1) the purpose of disclosure should be clear; 2) the patient and family members need to be able to accept the diagnosis; 3 ) the nature of the relationship between medical professionals and patient and family should be considered, and 4) psychological support should be provided to the patient after disclosure. These recommendations have clear symbolic significance, though it is unclear what impact they have had on clinical practice.

\section{Case analysis}

1) FAMILY IN DECISION MAKING

This case illustrates the common pattern of "family consent for disclosure" of the cancer diagnosis in Japan. A physician often discusses the cancer diagnosis with the family prior to discussion of the diagnosis with the patient. ${ }^{91}$ It is commonly held that the family best knows the patient's personality and the patient's ability to handle information about the cancer diagnosis. Physicians and family members alike fear that the patient will be shocked by a diagnosis of terminal illness and lose hope. Since the family's support is essential for the care of the patient, physicians feel it is best to seek the family's consent for disclosing a diagnosis of cancer to the patient, even when the patient is competent. This pattern of consultation emphasises the particular importance of the family's wishes and their influence on decision making. ${ }^{25}$ If the physician thinks the patient should be told, frequently he or she will try to persuade the family to disclose the cancer diagnosis to the patient. ${ }^{21}$ Ultimately, without the family's consent for disclosure, a large number of physicians feel there is no alternative but not to disclose the diagnosis fully and to deceive the patient if asked directly. ${ }^{21}$

Tomoaki Tsuchida sees the power of the family in disclosure decisions as a reflection of the family's role in Japanese society in general. In a comparison with cancer disclosure in the US, he states:

"For the American, it is not only a right to exercise control over one's own destiny, but also, one's duty. Death and life are one's own private concern. The Japanese, in contrast, have lived for centuries in a highly integrated and contextualized society where even life and death have to be seen as a family affair - if not the affair of the community as a whole - as much as the affair of the particular individual. 
Without the consent of the family, a doctor is not expected to inform a patient of a fatal illness or even to undertake serious surgery, much less organ transplant."26

While the family's role in clinical decision making for patients in Japanese society is important, there is an obvious risk of overemphasising it. Societal changes such as industrialisation have had an enormous impact on the family and have resulted in less community cohesion. ${ }^{26}$ There have always been patients without family members, and the number of nuclear families in Japan is increasing. Further, the media has taken an active role in promoting disclosure of the diagnosis and it has become common to disclose information about an early stage cancer diagnosis directly to the patient. ${ }^{22}{ }^{27}$ Finally, there are cases when family decision making conflicts with the best interests of the patient. Under such circumstances, intervention by doctors or others may be necessary.

\section{2) COMMUNICATION STYLE}

This case also delineates unique communication patterns about the cancer diagnosis in Japan. First, the physician initially communicated the cancer diagnosis to the family. Second, the physician gave the patient information about her condition which was ambiguous and, at face value, inaccurate. The physician here said: "You don't have any cancer yet, but if we don't treat you, it will progress to a cancer". Not only did the patient indeed have cancer, but also she had an incurable cancer with a dismal prognosis. Some would object that conveying incorrect information to a patient hardly amounts to disclosure. In the context of Japanese language and culture, however, we assert that there is much more being communicated than contained in the literal words. The fact that the treating physician even mentioned the word "cancer" sends an implicit message to the patient that there is a very serious problem. Readers of Japanese would know intuitively that the patient would sense that she might have cancer because the physician actually used the word "cancer". In this way, he ambiguously disclosed the truth to the patient.

Further, the way the message was presented to the patient could be interpreted in more than one way. One interpretation, a literal interpretation of what was said, is that the patient is affected by a premalignant condition. A second interpretation is that she indeed has cancer, but that her physician, in accordance with the family's request, did not want to shock her with overt disclosure of the cancer diagnosis and wished to leave her with the option of hope. In this way, the patient has the option of either a positive interpretation that she has a premalignant condition that is treatable, or negative interpretation that she does in fact have cancer and that her physician is trying to be sensi tive to the fact that she has an incurable condition and does not want to force her to hear the truth Similarly, the physician considered the family'佂 preference for the patient not to be told the diag $\mathrm{o}$ nosis as he never explicitly told the patient she had? cancer.

The pattern of communication in this case reflects an understanding that many Japanes people are accustomed to and commonly prefe $\bar{B}$ ambiguity. There is no need to be direct abouf such a delicate matter, and in fact, being too directr is often considered insensitive and cruel. Though it appears that the physician actively deceived the patient, it could be interpreted that he was offering to tell her the details of her case, but in aculturally sensitive way. His ambiguous presenta tion of the information to her could be regarded as a sombre overture to discussion of the case $\frac{\text { क }}{4}$ although only the strong-willed would push too open the crack in the door. The ambiguous preste entation in this sense may represent a Japanese form of "offering truth". ${ }^{28}$ Discussions about can cer disclosure often become stuck on whether the diagnosis was or wasn't told. This case illustrate that the debate ought to focus as much attention on the process of communication in the course of providing sensitive medical information to paЭ tients.

3) ADVANCE DIRECTIVES FOR CANCER DISCLOSURE While we believe many Japanese people would agree with our interpretation of the communica $\stackrel{3}{3}$ tion patterns in the above case, the process o\$ second-guessing may require the use of mentaR energy, both for the physician who has to conveg information to the patient, and for the patient and the family who must second-guess the infor mation communicated by the physician. Arf advance directive for cancer disclosure max̌ reduce the mental energy required in this indirec: process. In common parlance, an advance direc ${ }^{\omega}$ tive is obtained with the intent of directing decision making when the patient loses decision making capacity. In contrast, we define the "advance directive for disclosure" as a verbal or written declaration from the patient that indicate. his or her preference for disclosure of informations in the event cancer is diagnosed, even if the patien $\overrightarrow{0}$ has decision making capacity at the time of diago nosis. This kind of approach for resolving the dilemma about whether to disclose a cancer diag응 nosis in Japan has been previously proposed. ${ }^{29}{ }^{30}$ 
This case presents one example of an oral advance directive for cancer disclosure. The patient expressed her preference verbally to the family at an unknown time in the past when she was healthy. She indicated in advance her preference not to be told a cancer diagnosis. While there remains some need for second guessing about whether her preferences might have changed, we would assert that her advance directive for disclosure provided a tangible coordinate for directing decision making. This illustrates its appeal: the patient had input into the decision, but was not forced to confront directly the implications of her cancer diagnosis.

Clearly, interest in the topic of advance directives in Japan has increased. The Japan Society for Dying with Dignity, established in 1976, has witnessed a steady rise in membership to more than 90,000 members (about $0.08 \%$ of the total Japanese population) as of May 1999. Moreover, recent empirical research illustrates the degree to which awareness of advance directives is spreading. ${ }^{31}$ In one survey of 210 healthy male subjects ( $94.2 \%$ response rate) who visited two urban general hospitals for physical check-ups, $80.5 \%$ of respondents knew the term "living will" and wanted to express their preferences for future medical care. Regarding disclosure of diagnosis and prognosis, $79.7 \%$ indicated a desire to designate their preferences in advance. Most $(87.8 \%)$ answered they would give extensive leeway to surrogates to override their preferences and did not feel the necessity for detailed, concrete directives. Sixty-two per cent answered that oral statements were enough, while $29.7 \%$ thought that written documents were necessary. In regard to their preferred surrogate decision maker, $80.2 \%$ answered they would designate family or relatives, in most cases a spouse. Of the 38 respondents (18.1\%) who did not want to express their preferences in advance, the most common reasons given for not wanting to complete a living will included: 1) psychological resistance to talk about death and dying; 2) difficulty imagining future circumstances, a factor that highlights one of the theoretical limitations of advance directives, and 3) their preference for entrusting family to make the decision.

Although many Japanese people have heard of advance directives in the form of a living will, less than $1 \%$ of the population has completed one in written form. ${ }^{31}$ Neither advance directives nor power of attorney have been afforded legal status in Japan through legislation. Given the longstanding tradition of ambiguity in Japanese relationships, it is unclear whether written advance directives can be expected to become a widely uti- lised mechanism for the expression of patient autonomy as conceptualised in modern medical ethics for cancer disclosure or end-of-life treatments.

\section{4) UNIVERSALITY OF AUTONOMY}

The North American bioethicist Edmund Pellegrino argues that autonomy or "something close to autonomy" is a universal principle and not just a "cultural artifact". ${ }^{32} \mathrm{He}$ argues that "... the democratic ideals that lie behind the contemporary North American concept of autonomy will spread and that something close to it will be the choice of many individuals in other countries as well". He suggests that a nation can enjoy the benefits of medical progress only by dealing constructively with the conflict between traditional values and modern medical progress.

Yet when considering this issue in the international context, the term "autonomy" should be used carefully since it is not a concept with only one meaning. ${ }^{33}$ Pellegrino does not specify whether his notion of a North American concept of autonomy refers to the definition of autonomy or the degree of exercise of autonomy, or both. Surbone's remark that autonomy is often synonymous with isolation in Italy illustrates that the exercise of autonomy differs in Italy and North America, even though the definition may be very similar.

If autonomy and what it represents is to be viewed as a truly universal principle then it is plausible that the North American paradigm is only one version of it. There is no necessity for every country to follow the practice of autonomy in all of its details in a fashion identical to that found in North America. Pellegrino himself seems to be advocating autonomy in general, and not advocating exclusively the North American version of autonomy as a universal principle since it is so enmeshed with facets of Western culture such as science, ethics, and politics. He states:

"The dominant characteristics of Western science, ethics, and politics are mutually supportive: Western science is empirical and experimental, pursuing objectivity and quantification of experience. Ultimately, it attempts to control nature to the greatest extent possible. Western ethics is analytical, rationalistic, dialectical, and often secular in spirit. Western politics is liberal, democratic, individualistic, and law-governed. Western science, ethics, and politics provide an environment that gives rise to, and sustains, the use of complex medical technologies. As a result, it is difficult to divorce medical knowledge and the benefits it 
offers from the Western cultural and ethical milieu that supports and sustains it." 34

The fundamental question thus becomes: "What will 'something close to autonomy' look like in other countries?" Specifically, does this case inform us about what "something close to autonomy" looks like in Japan?

Some might assert that the process of ambiguous disclosure is not consistent with the meaning of "respect for something close to autonomy". The disclosure process in our case did not include a frank dialogue between the doctor and the patient, which is commonly held to be necessary in most contemporary articulations of the operation of autonomy. However, one cannot claim that this patient's wishes were not considered. She expressed her preferences in advance and these preferences were confirmed by her treating physician through ambiguous disclosure. She could interpret the message as being that she had cancer, or that she didn't have cancer. Since the physician didn't explicitly tell her she had cancer, her preference not to be told was partly respected. At that time she had the opportunity to ask questions if she so desired, contrary to her previously expressed wish not to be told. The patient's family played a critical role in facilitating her wishes and supporting her until the time of her death. Thus, in the context of Japanese society it can be argued that "something close to autonomy" was respected.

As this case reveals, concepts may exist in Japan that are similar to autonomy in the broad sense, even though the concept of autonomy has not been developed to fit the contemporary Japanese context. Higuchi proposes that a modified version of autonomy could be used in Japan..$^{35} \mathrm{He}$ suggests "autonomy" could be achieved through a process similar to that of "self-determination". However, his provocative analysis doesn't provide sufficient detail, lacking such important items as a definition of autonomy and an accounting for its implementation in clinical settings. Clearly, there is a need to develop a new or expanded formulation of autonomy for contemporary Japan.

\section{Implications}

While the "universal" concept of autonomy is regarded as accommodating cultural pluralism, practical problems may arise in transcultural settings among people from culturally diverse backgrounds who may not be familiar with or accustomed to exercising autonomy. ${ }^{36}{ }^{37}$ Learning how to deal with diverse patients in a culturally sensitive manner is an urgent item on the medical ethics agenda. Several deliberative discussions seek culturally sensitive approaches for clinica settings. ${ }^{283839}$ Non-verbal communication an $\bar{\phi}$ ambiguous disclosure as in the current case are known to occur in clinical practice outside of Japan as well. ${ }^{4041}$

This case discussion provides a window fo온 understanding the magnitude of the ethics researc agenda for contemporary Japan. As in other coun tries without a tradition of cancer disclosure, compelling issue remains how to define "somethin $\overrightarrow{\mathbf{g}}$ close to autonomy" in a way that is consistent with indigenous moral values, and does not threatego cultural identity. Criticism of the Anglo-American philosophy as ethnocentric alone will not help solv the real-life ethical dilemmas of non-Westers countries. Although autonomy may be construed as a universal principle, the definition or exercise of "something close to autonomy" in other countries may ultimately be very different. We posit the need for more exploration of the meaning and applicabil ity of autonomy in Japan and other similarly situated nations.

We believe autonomy or "something close tӪ autonomy" as a concept should be furthes developed by taking into account the cultural context. Further analysis of cancer disclosure io Japan has relevance for countries or culture which are adopting modern medical sciences but where disclosure of cancer is not a social normo Finally, we believe improved understanding of cultural differences, communication styles, an $\bar{\Phi}$ alternative accepted roles of the family in medical decision making will further the understanding modern medical ethics in pluralistic societies.

\section{Acknowledgement}

This research was made possible in part by Grant-In-Aid for Scientific Research, no@ 10557238 and 09672297 by the Ministry of Edu? cation, Science, Sports, and Culture, Japan, (AA) the Robert Wood Johnson Clinical Scholar 8 Program (MF); and the Japan-United States. Educational Commission (the Fulbright Pro\% gram) (TE).

Akira Akabayashi, MD, PhD, is Assistant Professor ifi the School of Health Science and Nursing, The University of Tokyo, Japan. Michael D Fetters, Mt $M A, M P H$, is Assistant Professor in the Department of Family Medicine, University of Michigan, Anin Arbor, Michigan, USA. Todd S Elwyn, MD, $\mathcal{F D}$, Resident in the Department of Psychiatry, Universit of Hawaii, Honolulu, Hawaii, USA. Correspondin $\overline{\mathrm{D}}$ address: Dr Akira Akabayashi, School of Health Scio ence and Nursing, University of Tokyo, 7-3-1 Hongo Bunkyo-ku, Tokyo 113-0033, fapan. Tel: 81-3-58410 3488, Fax: 81-3-5684-6083. 


\section{References}

1 Brown $\mathrm{KH}$, Jameton A. Information disclosure: attitude toward truth-telling. In: Reich WT, ed. Encyclopedia of bioethics. New York: Simon \& Schuster, Macmillian, 1995: 1221-5.

2 Jameton A. Information disclosure: II. Ethical issues. See reference $1: 1225-32$

3 Surbone A. Truth telling to the patient. Fournal of the American Medical Association 1992; 268,13: 1661-2.

4 Estape J, Palombo H, Hernandez E, et al. Cancer diagnosis disclosure in a Spanish hospital. Annals of Oncology 1992;3:451-4.

5 Dalla-Vorgia P, Katsouyanni K, Garanis TN, Touloumi G, Drogari P, Koutselinis A. Attitudes of a Mediterranean population to the truth-telling issue. Fournal of Medical Ethics 1992;18: 67-74.

6 Thomsen OO, Wulff HR, Martin A, Singer PA. What do gastroenterologists in Europe tell cancer patients? Lancet 1993; 341:473-6.

7 Harrison A, Al-Saadi AMH, Al-Kaabi ASO, et al. Should doctors inform terminally ill patients? The opinions of nationals and doctors in the United Arab Emirates. Fournal of Medical Ethics 1997;23:101-7.

8 Holland JC, Geary N, Marchini A, Tross S. An international survey of physician attitudes and practice in regard to revealing the diagnosis of cancer. Cancer Investigation 1987;5,2:151-4.

9 Long SO, Long BD. Curable cancers and fatal ulcers, attitudes toward cancer in Japan. Social Science and Medicine 1982;16: 2101-8.

10 Okayasu M. Presenting a diagnosis of cancer to patients in Japan. Nihon University fournal of Medicine 1988;30:259-67.

11 Morioka Y. Informed consent and truth telling to cancer patients. Gastroenterologica faponica 1991;26,6:789-92.

12 Mizushima Y, Kashii T, Hoshino K, et al. A survey regarding the disclosure of the diagnosis of cancer in Toyama prefecture, Japan. Fapanese fournal of Medicine 1990;29,2: 146-55.

$13 \mathrm{Li} \mathrm{S}$, Chou J-L. Communication with the cancer patient in China. In: Surbone A, Zwitter M, eds. Communication with the cancer patient - information and truth. New York: New York Academy of Sciences, 1997:243-8.

14 Burn G. From paper to practice: quality of life in a developing country - the challenges that face us. In: Surbone A, Zwitter M, eds. Communication with the cancer patient - information and truth. New York: New York Academy of Sciences, 1997: 249-60.

15 Matsuoka J. Whether cancer patients should be informed of their disease (in Japanese). Fournal of Fapanese Cancer Therapy 1970;5:114.

16 Yokoo E, Maruoka Y, Rokukawa K, Hidaka T, Sangu Y, Ogiuchi H. A questionnaire survey on whether the presence of a cancer should be revealed (in Japanese). Fapanese fournal of Cancer Clinics 1991;37,14:1710-15.

17 Sato I, Tazawa H, Fukaura A, et al. The questionnaire about informing of cancer the patients in Showa University Hospital (in Japanese). Proceedings of the Congress of the Japan Society for Cancer Therapy 1994;29:1677-85.

18 Tanida N. Japanese attitudes towards truth disclosure in cancer. Scandinavian fournal of Social Medicine 1994;22,1:50-7.

19 Osaki Y. Telling cancer patients of the diagnosis in our hospital (in Japanese). Nisseki Igaku 1991;43:187-91.

20 Kobayashi K, Kawasaki C, Yajima C, et al. The investigation of telling the truth to lung cancer patients (in Japanese). The fournal of the Japan Society for Cancer Therapy 1994;29:1001-9.

21 Hashimoto N. Disclosure of the cancer diagnosis (in Japanese). Fournal of the Fapan Medical Association 1995;113:937-42.

22 Elwyn TS, Fetters MD, Gorenflo DW, Tsuda T. Cancer disclosure in Japan: historical comparisons, current practices. Social Science and Medicine 1998;46:1151-63.
23 Ministry of Health and Welfare. Fiscal year 1994 report on the socioeconomic survey of vital statistics: terminal care (in Japanese). Tokyo: Statistics and Information Department, Minister's Secretariat, 1994.

24 Ministry of Health and Welfare and the Japan Medical Association. Manual of terminal care (in Japanese). Tokyo: Chuo-Hoki, 1989.

25 Fetters MD. The family in medical decision making: Japanese perspectives. Fournal of Clinical Ethics 1998;9:143-57.

26 Tsuchida T. From ethos to ethics: Japanese views on life and death. In: Institute of Medical Humanities, Kitasato University School of Medicine, ed. Toward a new replenishment of medical education and hospital service. Tokyo: Shinzan sha, 1992: 319-25.

27 Oga H, Horie K, Yafune H, Sata N, Sata F. Truth telling of disease name and stage in advanced gastrointestinal cancer - the psychological effect of prognosis on surgeons at university hospital (in Japanese). Fapanese fournal of Cancer Clinics 1997; 43,12:1477-83.

28 Freedman B. Offering truth: one ethical approach to the uninformed cancer patient. Archives of Internal Medicine 1993;153: 572-6.

29 Asai A. Should physicians tell patients the truth? Western fournal of Medicine 1995;163: 36-9.

30 Hoshino K. Informed consent: six proposals that fit Japan (in Japanese). Tokyo: Maruzen, 1997.

31 Akabayashi A, Kai I, Itoh K, Tsukui K. The acceptability of advance directives in Japanese society - a questionnaire study for healthy people in physical check-up settings (in Japanese) Fournal of Fapan Association for Bioethics 1997;7,1:31-40.

32 Pellegrino ED. Is truth telling to the patient a cultural artifact? [editorial]. Fournal of the American Medical Association 1992; 268,13:1734-5

33 Beauchamp TL, Childress JF. Principles of biomedical ethics [4th ed]. New York: Oxford University Press, 1994: 120-88.

34 Pellegrino ED. Prologue: intersections of western biomedical ethics and world culture: problematic and possibility. In Pellegrino ED, Mazzarella P, Corsi P, eds. Transcultural dimensions in medical ethics. Frederick, Maryland: University Publishing Group, 1992: 13-19.

35 Higuchi $\mathrm{N}$. The patient's right to know of a cancer diagnosis: a comparison of Japanese paternalism and American selfdetermination. Washburn Law fournal 1992;31,3:455-73.

36 Galanti G. Caring for patients from different cultures - case studies from American hospitals. Philadelphia: University of Pennsylvania Press, 1991.

37 Giger J, Davidhizar R. Transcultural nursing [2nd ed]. St. Louis: Mosby, 1995.

38 Good M, Good B, Schaffer C, Lind S. American oncology and the discourse on hope. Culture, Medicine and Psychiatry 1990;14:59-79.

39 Orr RD, Marshall PA, Osborn J. Cross-cultural considerations in clinical ethics consultations. Archives of Family Medicine 1995;4:159-64.

40 Novack D, Detering B, Arnold R, Forrow L, M L, Pezzullo J. Physicians' attitudes towards using deception to resolve difficult ethical problems. Fournal of the American Medical Association 1989;261,20:2980-5.

41 Miyaji $N$. The power of compassion: truth-telling among American doctors in the care of dying patients. Social Science and Medicine 1993;36,3:249-64. 